\title{
A PROPERTY FOR INVERSES IN A PARTIALLY ORDERED LINEAR ALGEBRA
}

BY

\author{
TAEN-YU DAI AND RALPH DEMARR
}

\begin{abstract}
We consider a Dedekind $\sigma$-complete partially ordered linear algebra $A$ which has the following property: if $x \in A$ and $1<x$, then $-u \leqslant x^{-1}$, where $u=u^{2}$. This property is used to show that $A$ must be commutative. We also show that $A$ is the direct sum of two algebras, each of which behaves like an algebra of real-valued functions.
\end{abstract}

1. Introduction and definitions. In his memoir [7] R. V. Kadison discusses various characterizations of an algebra of continuous real-valued functions on a compact Hausdorff space. In particular, $\S 3$ is devoted to characterizing an ordered algebra as an algebra of real-valued functions (the algebra theorem of $\mathrm{M}$. $\mathrm{H}$. Stone). In [2] and [3] the authors discuss various ways of characterizing a partially ordered linear algebra (pola) as an algebra of real-valued functions. The purpose of this paper is to characterize a pola having a special property for inverses. The motivation for this property comes from the following example. Let $\widetilde{B}$ be the pola of all continuous real-valued functions defined on $[0,1]$, where the algebraic operations and the partial order are defined pointwise. It is well known that $\widetilde{B}$ has the Archimedean property but is not Dedekind $\sigma$-complete (definitions below). We may embed $\widetilde{B}$ in a Dedekind $\sigma$-complete pola $A$ as follows. Define $A=\{\tilde{x}, \alpha): \tilde{x} \in \widetilde{B}$ and $\alpha$ is real $\}$. If the algebraic operations are defined componentwise, then $A$ is a real linear algebra. The partial order in $A$ is defined as follows. Take $x=(\tilde{x}, \alpha) \in A$ and $y=(\tilde{y}, \beta) \in A$. We write $x \leqslant y$ if and only if $0 \leqslant \tilde{y}(\tau)-\tilde{x}(\tau) \leqslant \beta-\alpha$ for all $\tau \in[0,1]$. It is easy to verify that $A$ is Dedekind $\sigma$-complete. See Example 1 for the details. Examples are given in $\S 5$.

Note that $B=\{(\widetilde{x}, 0): \tilde{x} \in \widetilde{B}\}$ is a subalgebra of $A$ which is algebraically (but not order) isomorphic to $\widetilde{B}$. However, we may introduce a new partial order in $B$ so that $B$ is also order isomorphic to $\widetilde{B}$ (see $\S 4$ ). This can be done abstractly by noting that $A$ has the following property. Take $u=(\tilde{0}, 1) \in A$ and note that $0 \leqslant u=u^{2}$. Now if $x \in A$ and $1 \leqslant x$, then $x$ has an inverse and $-u \leqslant x^{-1}$.

We now give the basic definitions needed in this paper. A pola (denoted by

Received by the editors September 10, 1974 and, in revised form, December 15, 1974. AMS (MOS) subject classifications (1970). Primary 06A70; Secondary 15A45.

Key words and phrases. Dedekind $\sigma$-complete partially ordered linear algebra, algebra of real-valued functions, $f$-ring, matrix inequalities. 
$A$ ) is a real linear associative algebra which is partially ordered so that it is a directed partially ordered linear space and $0 \leqslant x y$ whenever $x, y \in A, 0 \leqslant x$, $0 \leqslant y$. We also assume that $A$ has a multiplicative identity $1 \geqslant 0$. A Dedekind $\sigma$-complete pola (dsc-pola) $A$ is one having the property: if $x_{n} \in A, 0 \leqslant \cdots \leqslant$ $x_{2} \leqslant x_{1}$, then $\inf \left\{x_{n}\right\}$ exists. Order convergence is defined as usual. A dsc-pola $A$ has the Archimedean property: if $x, y \in A$ and $n x \leqslant y$ for every positive integer $n$, then $x \leqslant 0$. For more details and examples see the references.

2. Basic lemmas and theorems. In this paper we assume that $A$ is a dsc-pola (not necessarily commutative) which has the following property: if $x \in A$ and $1 \leqslant x$, then $x$ has an inverse and $-u \leqslant x^{-1}$, where $u \in A$ is a fixed element such that $0 \leqslant u=u^{2}$.

We put $e=1-u$ and note that $e=e^{2}$ and $e u=u e=0$. The first step is to show that $u$ commutes with every element of $A$.

LEMMA 2.1. If $w \in A, 0 \leqslant w$ and $w^{2}=0$, then $w=0$.

PROOF. Since $1 \leqslant 1+n w$ for every positive integer $n$, we have $-u \leqslant$ $(1+n w)^{-1}=1-n w$, so that $n w \leqslant 1+u$ for all $n$. From the Archimedean property we get $w \leqslant 0$. Hence, $w=0$.

LEMMA 2.2. If $0 \leqslant z \leqslant u$, then $u z=z u$.

Proof. Put $w=e z u$. Note that $0 \leqslant u-w=(u-w)^{2}$. Hence, $1 \leqslant 1+$ $n(u-w)$ for every positive integer $n$ so that $-u \leqslant[1+n(u-w)]^{-1}=1-$ $n(n+1)^{-1}(u-w)$, which gives $-(u-w) \leqslant(n+1)(1+w)$ for all $n$. Thus, $0 \leqslant 1+w$ (from the Archimedean property). Since $w^{2}=0$, we get $0 \leqslant(1+w)^{n}$ $=1+n w$ for every positive integer $n$ so that $-n w \leqslant 1$ for all $n$. Using the Archimedean property again, we get $0 \leqslant w$. From Lemma 2.1 we get $w=e z u=0$.

We may repeat the above argument for the element uze to show that uze $=$ 0. Hence, $u z=u z(u+e)=u z u=(u+e) z u=z u$.

LEMMA 2.3. If $-\beta u \leqslant x \leqslant \beta u$ for some real number $\beta \geqslant 0$, then $x u=u x$.

Proof. This lemma follows directly from Lemma 2.2.

LEMMA 2.4. If $0 \leqslant y \leqslant z$ and $y^{n} \leqslant n z$ for every positive integer $n$, then $y \leqslant 1+u$.

PROOF. If $0 \leqslant \lambda<1$, then $\sum_{k=1}^{\infty}(\lambda y)^{k} \leqslant \Sigma_{k=1}^{\infty} \lambda^{k}(k z)<\infty$. Hence, $1 \leqslant$ $(1-\lambda y)^{-1}$; see Theorem I.6.1 of [2]. Therefore, $-u \leqslant 1-\lambda y$ or $\lambda y \leqslant 1+u$ for all $\lambda<1$. From the Archimedean property we get $y \leqslant 1+u$.

LEMMA 2.5. If $1 \leqslant x$, then $-u \leqslant x^{-1} \leqslant 1+u(x-1)$ and $-u \leqslant x^{-1} \leqslant$ $1+(x-1) u$. 
PROOF. The basic property of this paper asserts that $-u \leqslant x^{-1}$. The other inequalities follow directly from $0 \leqslant\left(x^{-1}+u\right)(x-1)$ and $0 \leqslant(x-1)\left(x^{-1}+u\right)$.

LEMMA 2.6. If $1 \leqslant y$ and $y u=u y u$ (or $u y=u y u$ ), then $y u=u y$.

Proof. From Lemma 2.5 we get $-u \leqslant y^{-1} \leqslant 1+(y-1) u$ or $0 \leqslant u+$ $y^{-1} \leqslant 1+y u$. We now show by mathematical induction that $0 \leqslant\left(u+y^{-1}\right)^{n}$ $\leqslant 1+3^{n}(u+y u)$ for every positive integer $n$. This is easy to verify for $n=1$. If the above inequalities are true for $n=k$, then

$$
\begin{aligned}
0 \leqslant\left(u+y^{-1}\right)^{k+1} & \leqslant\left(u+y^{-1}\right)\left[1+3^{k}(u+y u)\right] \\
& \leqslant 1+y u+3^{k}(u+y u+y u+u) \leqslant 1+3^{k+1}(u+y u),
\end{aligned}
$$

where we have used the fact that $u y u=y u, y^{-1} u+u \leqslant u+y u$ and other elementary inequalities. This completes the induction.

It follows that $\left[3^{-1}\left(u+y^{-1}\right)\right]^{n} \leqslant 1+2 y u$ for every positive integer $n$. Using Lemma 2.4, we get $0 \leqslant u+y^{-1} \leqslant 3(1+u)$. Therefore, $-5 u \leqslant u y^{-1} \leqslant$ $5 u$ and $-5 u \leqslant y^{-1} u \leqslant 5 u$. From Lemma 2.3 we get $u y^{-1}=u y^{-1} u$ and $u y^{-1} u$ $=y^{-1} u$. Hence, $u y^{-1}=y^{-1} u$ so that $y u=u y$.

To prove this lemma when we assume that $u y=u y u$ one starts with the inequality $0 \leqslant u+y^{-1} \leqslant 1+u y$ (from Lemma 2.5) and then shows (as above) that $0 \leqslant\left(u+y^{-1}\right)^{n} \leqslant 1+3^{n}(u+u y)$ for all $n$. Hence, $u+y^{-1} \leqslant 3(1+u)$ and the rest of the proof follows as above.

LEMMA 2.7. If $1 \leqslant x$, then $u x=x u$.

Proof. Define $y_{1}=1+u(x-1)$ and $y_{2}=1+(x-1) u$. Note that $1 \leqslant y_{1}, 1 \leqslant y_{2}$ and $y_{1} u=u y_{1} u=u x u=u y_{2} u=u y_{2}$. From Lemma 2.6 we get $u y_{1}=y_{1} u=u y_{2}=y_{2} u$. But $u x=u y_{1}=y_{2} u=x u$.

THEOREM 2.8. If $z \in A$, then $u z=z u$.

Proof. Since $A$ is directed, we may write $z=x_{1}-x_{2}$, where $1 \leqslant x_{1}$ and $1 \leqslant x_{2}$. The theorem follows from Lemma 2.7.

We may now define $B=\{x: e x=x\}$ and $N=\{x: u x=x\}$. It is easy to see that $B$ and $N$ are real linear algebras and that $A$ is the direct sum of $B$ and $N$. The remaining lemmas will be used later to describe the various properties of $B$ and $N$.

LEMMA 2.9. If $1 \leqslant x$, then $-5 u \leqslant u x^{-1} \leqslant 5 u$.

Proof. Since $u x=x u$, we have $x u=u x u$. We now refer to the proof of Lemma 2.6 to get $-5 u \leqslant u x^{-1} \leqslant 5 u$.

LEMmA 2.10. If $x \in A, t \in N$ and both $x$ and $x+t$ have inverses, then $e x^{-1}=e(x+t)^{-1}$. 
Proof. Since $t \in N$, we have $e t=0$. Hence, $e x=e(x+t)$ and the result follows easily, but one must use the fact that $e x=x e$.

LEMMA 2.11. If $1 \leqslant x$, then $-u \leqslant e x^{-1}$.

Proof. Since $1 \leqslant x(1+n u)$ for every positive integer $n$, we have $-u \leqslant$ $(1+n u)^{-1} x^{-1}=\left[1-n(n+1)^{-1} u\right] x^{-1}=\left[e+(n+1)^{-1} u\right] x^{-1}$ for all $n$. From the Archimedean property it follows that $-u \leqslant e x^{-1}$.

LEMMA 2.12. If $1 \leqslant x$, then $e x^{-1} \leqslant 1$.

Proof. Using Lemma 2.11, we get $0 \leqslant\left(u+e x^{-1}\right)(x-1)$, from which it follows that $0 \leqslant u+e x^{-1} \leqslant e+u x$. We now show by mathematical induction that $0 \leqslant\left(u+e x^{-1}\right)^{n} \leqslant e+n(u x)$ for all $n$. The inequalities are clearly true for $n=1$. If they are true for $n=k$, then

$$
\begin{aligned}
0 \leqslant\left(u+e x^{-1}\right)^{k+1} & \leqslant(e+k u x)\left(u+e x^{-1}\right)=e x^{-1}+k u x \\
& \leqslant u+e x^{-1}+k u x \leqslant e+u x+k u x=e+(k+1) u x .
\end{aligned}
$$

This completes the induction.

Since $e \leqslant 1$, we get $0 \leqslant\left(u+e x^{-1}\right)^{n} \leqslant n(1+u x)$ for all $n$. From Lemma 2.4 we get $u+e x^{-1} \leqslant 1+u$ so that $e x^{-1} \leqslant 1$.

LEMMA 2.13. If $1 \leqslant x \leqslant y$, then $-u \leqslant e x y^{-1} \leqslant 1$.

Proof. Since $-u \leqslant x^{-1}$, we get $0 \leqslant(y-x)\left(x^{-1}+u\right)$, from which $1 \leqslant$ $[y+u(y-x) x] x^{-1}$. From Lemmas 2.11 and 2.12 we get $-u \leqslant e x[y+u(y-x) x]^{-1}$ $\leqslant 1$. Since $u(y-x) x \in N$, we may use Lemma 2.10 to get $-u \leqslant e x y^{-1} \leqslant 1$.

LEMmA 2.14. If $0 \leqslant z \leqslant 1+t$, where $t \in N$ and $0 \leqslant t$, then $-u \leqslant e z \leqslant 1$.

Proof. Since $1 \leqslant 1+n z \leqslant 1+n(1+t)$ for every positive integer $n$, we can use Lemmas 2.10 and 2.13 to get $-u \leqslant e(1+n z)[(n+1) 1+n t]^{-1}=$ $(n+1)^{-1} e(1+n z) \leqslant 1$. From the Archimedean property we get $-u \leqslant e z \leqslant 1$.

LEMmA 2.15. If $a \in B, s \in N$ and $-s \leqslant a \leqslant s$, then $a=0$. (Note that $e a=a$ and $e s=0$.)

Proof. It is clear that $0 \leqslant s+a \leqslant 2 s$. Therefore, $0 \leqslant n(s+a) \leqslant 1+2 n s$ for every positive integer $n$. Hence, from Lemma 2.14 we get $-u \leqslant n e(s+a)=$ $n a \leqslant 1$ for all $n$. From the Archimedean property we get $a=0$.

LEMMA 2.16. If $0 \leqslant x \leqslant y$ and $1 \leqslant y$, then $-u \leqslant e x y^{-1} \leqslant 1$.

Proof. Since $0 \leqslant y^{-1}+u$, we get $0 \leqslant x\left(y^{-1}+u\right) \leqslant y\left(y^{-1}+u\right)=1+y u$. Since $y u \in N$ and $0 \leqslant y u$, we may use Lemma 2.14 to get $-u \leqslant \operatorname{ex}\left(y^{-1}+u\right)=$ $e x y^{-1} \leqslant 1$.

LEMMA 2.17. If $0 \leqslant z$ and $e z \leqslant x$, where $1 \leqslant x$, then $0 \leqslant e z+u x$. 
Proof. Since $0 \leqslant z=e z+u z \leqslant x+u z$ and $1 \leqslant x+u z$, we may use Lemma 2.16 to get $-u \leqslant e z(x+u z)^{-1} \leqslant 1$. Since $u z \in N$, it follows from Lemma 2.10 that $-u \leqslant e z x^{-1}$. Hence, $-u x \leqslant e z$ or $0 \leqslant e z+u x$.

3. The structure of $N$. Since $0 \leqslant u$, it is clear that $N$ is a directed pola and that $u$ is the identity for $N$. We will see that the structure of $N$ is actually characterized in [2] but first we need two lemmas.

LEMMA 3.1. $N$ is order-convex.

Proof. We need only show that if $-t \leqslant x \leqslant t$ and $t \in N$, then $x \in N$ (recall that $N$ is directed). Since $x=e x+u x$ and $-t \leqslant u x \leqslant t$, we get $-2 t \leqslant$ $e x \leqslant 2 t$. From Lemma 2.15 we obtain $e x=0$ so that $x=u x \in N$.

LEMMA 3.2. $N$ is closed with respect to order convergence.

Proof. Let $\left\{x_{n}\right\}$ be a sequence of elements from $N$ such that $o$-lim $x_{n}=x$. Thus, for some element $z \in A$ we have $-z \leqslant x_{n} \leqslant z$ for all $n$. Hence, $-u z \leqslant$ $u x_{n}=x_{n} \leqslant u z$ for all $n$, which means $-u z \leqslant x \leqslant u z$. Since $u z \in N$ and $N$ is order-convex, we have $x \in N$.

These lemmas enable us to assert that $N$ is Dedekind $\sigma$-complete. Hence, $N$ is a dsc-pola with identity $u$. We may now apply the results of [2]. Note that in [2] the term "polac" is used instead of "dsc-pola". Let $N_{1}$ denote the functional part of $N$ as described in [2, p. 658].

THEOREM 3.3. $N=N_{1}$.

Proof. Take any $t \in N$ such that $u \leqslant t$. Put $x=e+t=1+(t-u)$ so that $1 \leqslant x$. From Lemma 2.9 we get $-5 u \leqslant u x^{-1} \leqslant 5 u$, which means that $u x^{-1} \in N_{1}$. Hence, $0 \leqslant\left(u x^{-1}\right)^{2}$. Since $u \leqslant t \leqslant t^{2}$ and $t^{2}\left(u x^{-1}\right)^{2}=\left(u x^{-1}\right)^{2} t^{2}$ $=u$, we see that $t^{2} \in N_{1}$. Since $N_{1}$ is order-convex, we can assert that $t \in N_{1}$. Since $N$ is directed, we get $N=N_{1}$.

In particular this means that $N$ is commutative. The reader is referred to [2] for a more detailed discussion.

4. The structure of $B$. In the special case that $u \leqslant 1$ we have $0 \leqslant e$ and one may show that $B=B_{1}=$ the functional part of $B$. In fact, one may show that $A=A_{1}=$ the functional part of $A$. We leave this as an exercise for the reader.

In general, we must introduce a new partial order in $B$. To do this we define $K=\{e z: 0 \leqslant z\} \subset B$. The reader may easily verify that $K$ is a generating cone in $B$ and that $K$ is closed with respect to multiplication.

Lemma 4.1. If $a \in K$ and $-a \in K$, then $a=0$.

Proof. Suppose $0 \leqslant y$ and $0 \leqslant z$ are such that $a=e y$ and $-a=e z$. Since 
$e y \leqslant y$ and $e z \leqslant z$, we get $-(y+z) \leqslant a \leqslant y+z$. But $e(y+z)=a-a=0$ so that $y+z=u(y+z) \in N$. From Lemma 2.15 we get $a=0$.

We may now define a partial order $\leqslant_{0}$ in $B$ as follows: for $b, c \in B$ we write $b \leqslant_{o} c$ if and only if $c-b \in K$. Lemma 4.1 is used to assert that $\leqslant_{o}$ is antisymmetric.

LEMMA 4.2. The real linear algebra $B$ with the partial order $\leqslant_{0}$ is a directed pola which has the Archimedean property.

Proof. It is easily verified that $B$ is a directed pola. Let us now assume that $b, c \in B$ and $n b \leqslant_{o} c$ for every positive integer $n$. Thus, for each $n$ we can find $z_{n} \in A$ such that $0 \leqslant z_{n}$ and $e\left(n z_{n}\right)=c-n b$. We may now take $y \in A$ so that $0 \leqslant y$ and $c \leqslant y$. Since $0 \leqslant y$ and $n \geqslant 1$, we get $e z_{n} \leqslant y-b$ for all $n$. Next select $x \in A$ so that $1 \leqslant x$ and $e z_{n} \leqslant y-b \leqslant x$. From Lemma 2.17 we get $0 \leqslant$ $e z_{n}+u x$ for all $n$. Therefore, $0 \leqslant n\left(e z_{n}+u x\right)=c+n(u x-b)$ for all $n$. Since the partial order $\leqslant$ in $A$ has the Archimedean property, we have $0 \leqslant u x-b$. This means that $e(u x-b)=-b \in K$. Hence, $b \leqslant_{0} 0$.

Lemma 4.3. If $f \in B$ and $e \leqslant_{o} f$, then there exists $g \in B$ such that $0 \leqslant_{o} g$ and $f g=g f=e$.

Proof. There exists $y \in A$ such that $0 \leqslant y$ and $e y=f-e$. Since $f=$ $e(1+y)$ and $1 \leqslant 1+y$, we have $f(1+y)^{-1}=(1+y)^{-1} f=e$ and $0 \leqslant u+$ $e(1+y)^{-1}$ from Lemma 2.11. If we put $g=e(1+y)^{-1}$, then it is easy to show that $g$ has the desired properties.

THEOREM 4.4. $B$ is commutative.

Proof. Define $F=\bigcup_{n=1}^{\infty}\left\{f \in B:-n e \leqslant_{0} f \leqslant_{0} n e\right\}$. It is clear that $F$ is an order-convex subalgebra of $B$ which has $e$ as an order unit. Also, $F$ has the Archimedean property. It is known that $F$ is isomorphic to an algebra of bounded real-valued functions; see [8, p. 255, Exercise 24]. This means that $F$ is commutative. If $a, b \in B$ and $e \leqslant_{o} a$ and $e \leqslant_{o} b$, then there exist elements $c, d \in B$ such that $0 \leqslant \leqslant_{o} c, 0 \leqslant d$ and $a c=c a=e=b d=d b$. But $0 \leqslant \leqslant_{o} e c=c \leqslant_{o} a c=e$ and $0 \leqslant_{o} e d=d \leqslant_{o} b d=e$, which means that $c \in F$ and $d \in F$. Thus, $c d=d c$ and it follows easily that $a b=b a$. Since $B$ is directed, it follows that $B$ is commutative.

From the above results it follows that $A$ is a commutative algebra. In this connection the authors recommend the very interesting paper of Jamison [6]

5. Examples. The basic example was described in the introduction. The reader should note that Example 4 is an important counterexample.

EXAMPLE 1. This example was described in the introduction. Here we wish to show that $A$ is Dedekind $\sigma$-complete. Let $x_{n} \in A$ be a sequence such that 
$0 \leqslant \cdots \leqslant x_{2} \leqslant x_{1}$. Now $x_{n}=\left(\tilde{x}_{n}, \lambda_{n}\right)$ and we must have $0 \leqslant \cdots \leqslant \lambda_{2} \leqslant \lambda_{1}$. Define $\mu=\inf \left\{\lambda_{n}\right\}$ and note that $0 \leqslant \tilde{x}_{n}(\tau)-\tilde{x}_{k}(\tau) \leqslant \lambda_{n}-\mu$ for all $\tau \in[0,1]$ and all $n \leqslant k$. Thus, the sequence $\left\{\tilde{x}_{n}\right\}$ of functions converges uniformly to a continuous function $\tilde{y}$. If we put $y=(\tilde{y}, \mu)$, then it is easy to show that $y=$ $\inf \left\{x_{n}\right\}$.

EXAMPLE 2. Let $\widetilde{B}$ be the real linear algebra of all continuous real-valued functions defined on the real line. Let

$$
\left.A=\left\{\tilde{x} ; \alpha_{1}, \alpha_{2}, \ldots\right): \tilde{x} \in \tilde{B} \text { and } \alpha_{n} \text { real for all } n\right\} .
$$

If the algebraic operations are defined componentwise, then $A$ is a real linear algebra. The partial order $\leqslant$ in $A$ is defined as follows: if $x=\left(\tilde{x} ; \alpha_{1}, \alpha_{2}, \ldots\right)$ and $y=\left(\tilde{y} ; \beta_{1}, \beta_{2}, \ldots\right)$, then $x \leqslant y$ if and only if $0 \leqslant \tilde{y}(\tau)-\tilde{x}(\tau) \leqslant \beta_{n}-\alpha_{n}$ for all $n$ and for all $\tau \in[-n, n]$. As in Example 1 we can show that $A$ is a dsc-pola. The reader should note that order convergence of sequence of elements from $A$ implies that the corresponding functions converge uniformly on every finite interval. The above idea can be generalized to any algebra $\widetilde{B}$ of functions on a locally compact space.

We may define $u=(\tilde{0} ; 1,1, \ldots)$ and then show that if $1 \leqslant x$, then $-u$ $\leqslant x^{-1}$. Note that in this case the elements of $B$ and $N$ need not be bounded functions.

EXAmple 3. Let $A$ be the set of all real-valued functions defined on the real line. If $x, y \in A$, we define $x=y$ and $x \leqslant y$ to mean that $x(\tau)=y(\tau)$ and $x(\tau) \leqslant y(\tau)$ for almost all $\tau$ (Lebesgue measure). Thus, $A$ is a dsc-pola, where the algebraic operations are defined pointwise (almost everywhere). Let $u \in A$ be the characteristic function of the set of positive real numbers. Thus, $u \leqslant 1$ so that $0 \leqslant e$. Note that the elements of $B$ and $N$ behave like real-valued functions but cannot be represented by real-valued functions which are defined everywhere on some set.

EXAMPLE 4. Let $A$ be the real linear algebra of all matrices of the form $x=\left[\begin{array}{ll}\alpha & \beta \\ 0 & \nu\end{array}\right]$. If we define $0 \leqslant x$ to mean that $0 \leqslant \alpha, 0 \leqslant \beta$ and $\alpha+\beta \leqslant \nu$, then $A$ is a dsc-pola. If we put $u=\left[\begin{array}{ll}0 & 1 \\ 0 & 2\end{array}\right]$, then it is a routine computation to show that if $1 \leqslant x$, then $-u \leqslant x^{-1}$ and that $-u$ is the best possible lower bound for inverses of elements $x$ such that $1 \leqslant x$. Note that $0 \leqslant 2 u=u^{2}$ and that $A$ is not commutative.

\section{REFERENCES}

1. G. Birkhoff, Lattice theory, 3rd ed., Amer. Math. Soc. Colloq. Publ., vol. 25, Amer. Math. Soc., Providence, R. I., 1967. MR 37 \#2638.

2. Taen-yu Dai, On some special classes of partially ordered linear algebras, J. Math. Anal. Appl. 40 (1972), 649-682.

3. Ralph DeMarr, $A$ class of partially ordered linear algebras, Proc. Amer. Math. Soc. 39 (1973), 255-260. MR 47 \#1716. 
4. D. W. Duboic, A note on David Harrison's theory of preprimes, Pacific J. Math. 21(1967), 15-19. MR 35 \#103.

5. - Second note on David Harrison's theory of preprimes, Pacific J. Math. 24 (1968), 57-68. MR 36 \#5049.

6. R. E. Jamison, Functional representation of algebraic intervals, Pacific J. Math. (to appear).

7. R. V. Kadison, A representation theory for commutative topological algebra, Mem. Amer. Math. Soc. No. 7 (1951). MR 13, 360.

8. H. H. Schaefer, Topological vector spaces, Macmillan, New York, 1966. MR 33 \#1689.

9. B. Z. Vulih, Introduction to the theory of partially ordered spaces, Fizmatgiz, Moscow, 1961; English transl., Wolters-Noordhoff, Groningen, 1967. MR 24 \#A3494; 37 \#121.

DEPARTMENT OF MATHEMATICS, YORK COLLEGE (CUNY), JAMAICA, NEW YORK 11451

DEPARTMENT OF MATHEMATICS, UNIVERSITY OF NEW MEXICO, ALBUQUERQUE, NEW MEXICO 87131 